\title{
1. Basic Surgical Techniques of Implant Placement
}

\author{
UCLA Dr. Thomas J. Han
}

A successful two stage endosseous implant therapy involves close integration of diagnostic, surgical and restorative skills. Especially in partially edentulous cases, understanding of restorative needs, anatomic considerations and esthetic requirements is essential, and intelligent hard and soft tissue management is necessary to achieve optimum success with minimum patient discomfort.

This is a clinically-oriented presentation designed to familiarize clinicians with some of the basic principles and surgical techniques of implant therapy.

Precise positioning of fixtures of proper length without damage to vital structure is critical. The methods by which periapical and panoramic radiograph can be utilized to accomplish this will be presented. The use of facial surface surgical stents during surgery will be shown.

Indications and utilization of tomograms and CT scans in presurgical treatment planning and assessment of fixture positioning will be discussed briefly.

During first and second stage surgical procedures, the proper soft tissue management will minimize surgical complications and morbidity, and contribute significantly to the long term success of implant therapy. The surgical techniques which will be covered in this presentation include:

First stage implant surgery : surgical flap design and tissue management technique.

Second stage surgery: flap management technique to allow for close adaptation of tissue, increase keratinized attached gingiva, and correct ridge deficiencies to improve esthetics.

Variations in flap design and management techniques in conjunction with guided tissue regeneration technique, when placing implants in extraction sockets, and in areas with high esthetic requirements.

Surgical managements of soft tissue complication after implant surgery. 